\title{
The Effect of Pregnancy on Disease Activity in Patients with Psoriatic Arthritis
}

\author{
Mark Berman, Devy Zisman, Jonathan Wollman, David Levartovsky, Eli Rimon, Ori Elkayam, \\ and Daphna Paran
}

\begin{abstract}
Objective. To evaluate the effect of pregnancy on disease activity in psoriatic arthritis (PsA).
Methods. This is a retrospective case series. Review of the medical files of all female patients followed at the PsA clinic of 2 medical centers identified those with at least 1 pregnancy during followup and 1 visit during or soon after pregnancy.

Results. Twenty-five women with PsA (out of 107 women of reproductive age followed up in our PsA clinics) and 35 pregnancies were enrolled. Thirty-three pregnancies resulted in live healthy babies. In the whole group, there was no significant change in disease activity throughout pregnancy, while in $16(48 \%)$ of pregnancies, patients worsened during the first postpartum year. In 15 out of 21 pregnancies, in which the women had been treated before conception with biologics, treatment was discontinued close to pregnancy or during the first trimester. Five of those 15 patients had been classified as having mild to severe PsA activity prior to pregnancy. That number increased to 8,9 , and 14 during the first and second trimesters and postpartum period, respectively. There was no significant change in degree of disease activity in 6 patients whose biologics were continued beyond the first trimester. Improvement in disease activity was observed during pregnancy among the nonbiologics-treated patients. Corticosteroids were initiated or the dosage was increased during 6 pregnancies, all involving patients whose biologics were stopped before pregnancy.

Conclusion. Continuation of biologics therapy was associated with a low level of disease activity and a low probability of flare during pregnancy. Stopping treatment with biologics before pregnancy is associated with flare during pregnancy and the postpartum period. (First Release September 15 2018; J Rheumatol 2018;45:1651-5; doi:10.3899/jrheum.171218)
\end{abstract}

Key Indexing Terms:

PSORIATIC ARTHRITIS

BIOLOGICAL THERAPY

PSORIASIS

TRIMESTER

PREGNANCY

Psoriasis is a common disease that affects $3-4 \%$ of the population. Psoriatic arthritis (PsA) develops in up to $30 \%$ of all patients with psoriasis ${ }^{1}$ and it often affects women of childbearing age. The effect on pregnancy of other inflammatory diseases, such as rheumatoid arthritis (RA) and systemic lupus erythematosus (SLE), has been extensively studied. RA improves during pregnancy in around 50-80\% of the women, with the improvement in disease activity starting early in the first trimester and often continuing

From the Rheumatology Department, and the Obstetrics and Gynecology Department, Tel Aviv Sourasky Medical Center, Tel Aviv; Rheumatology Unit, Carmel Medical Center, Haifa, Israel.

M. Berman, MD, Rheumatology Department, Tel Aviv Sourasky Medical Center; D. Zisman, MD, Rheumatology Unit, Carmel Medical Center;

J. Wollman, MD, Rheumatology Department, Tel Aviv Sourasky Medical

Center; D. Levartovsky, MD, Rheumatology Department, Tel Aviv

Sourasky Medical Center; E. Rimon, MD, Obstetrics and Gynecology

Department, Tel Aviv Sourasky Medical Center; O. Elkayam, MD,

Professor, Rheumatology Department, Tel Aviv Sourasky Medical Center;

D. Paran, MD, Rheumatology Department, Tel Aviv Sourasky Medical

Center. Dr. O. Elkayam and Dr. D. Paran contributed equally to this

manuscript.

Address correspondence to Dr. M. Berman, Rheumatology Department,

Tel Aviv Sourasky Medical Center, Weizmann 6, Tel Aviv, Israel.

E-mail:markbe@tlvmc.gov.il

Accepted for publication June 28, 2018. throughout the pregnancy. In about $90 \%$ of women, however, the RA flares during the postpartum period ${ }^{2,3}$. There may be exacerbations of SLE during pregnancy and the postpartum period in 25-60\% of cases, depending on the status of disease activity prior to pregnancy. Maternal mortality is reported to be 20 -fold higher aimong women with SLE who become pregnant, and there is a 2- to 4-fold increased rate of obstetric complications $\mathrm{s}^{4,5}$.

PsA, like RA, has also been reported to improve during pregnancy ${ }^{6}$, but unlike RA, there is a paucity of data on the influence of PsA on women's health status during pregnancy. One prospective study by Ostensen in 1988, prior to the biologics era, included 20 pregnancies in 15 women, and the findings suggested improvement of symptoms during pregnancy, and subsequent flare ${ }^{7}$. That study included patients with relatively low disease activity (oligoarthritis) and did not take into account patients with polyarthritis or axial disease. Importantly, significant changes have taken place in the treatment strategies of PsA since then, and a substantial number of patients are now being treated with biologic therapies. More recently, Polachek, et $a l^{8}$ reported on 29 women with PsA and 42 pregnancies; PsA improved or had stable low activity in $58.5 \%$ of those pregnancies.

Personal non-commercial use only. The Journal of Rheumatology Copyright (c) 2018. All rights reserved. 
During the postpartum period, 52.5\% had either improvement or stable low PsA activity, whereas $40 \%$ had either worsening or stable high disease activity. The skin activity during pregnancy either improved or stayed in a stable low state in $88.2 \%$, and there was worsening in $42.9 \%$ in the postpartum period. It was concluded that there was a trend toward a favorable course for the arthritis activity and a favorable course for the skin disease during pregnancy. Compared to the nonpregnant PsA women controls, the period of pregnancy had significant beneficial influence only on the skin and not on the joints ${ }^{8}$.

None of these studies addressed the issue of the effect of stopping or continuing biologics during pregnancy in patients with PsA. Therefore, we conducted a retrospective descriptive study with the aim of evaluating the effect of pregnancy on disease activity in PsA. The secondary endpoint of our study was to assess the effect of stopping or continuing biologics on disease activity of PsA during pregnancy and the postpartum period.

\section{MATERIALS AND METHODS}

Our study is a retrospective review of the medical files of female patients of reproductive age (18-49 yrs) followed at the Psoriatic Arthritis Clinic at the Tel Aviv Medical Center (Tel Aviv, Israel) and the Carmel Medical Center (Haifa, Israel). Ethics board approval was received from both Tel Aviv Medical Center (approval number 0041-15) and Carmel Medical Center (approval number 0048-16). According to the local guidelines, there was no need to obtain patient's written informed consent because the data collected were retrospective. Each file received a number and was anonymized. Patients with at least 1 pregnancy during their clinical followup and 1 visit within the first year after delivery were identified. Only the pregnancies that ended with the birth of a live and healthy baby without major congenital abnormalities were included in the study. This exclusion was due to the retrospective design of the study and the lack of solid data in the files concerning miscarriages. The following data on the women were retrieved: age; disease duration; pattern of PsA; disease activity before, during, and after pregnancy; and treatment (including intraarticular injections of corticosteroids). According to our center's policy, flares during pregnancies were treated with nonsteroidal antiinflammatory drugs and systemic or intraarticular corticosteroids.

The postpartum period was defined as the first year after delivery.

PsA activity was defined as follows: none (no active synovitis), mild (none to 1 joint involved), and moderate to severe ( 2 and more joints involved). The followup during and after pregnancy was classified as improvement, worsening, or stable, according to change in disease activity. A subanalysis of patients was also performed according to baseline treatment with biologic agents versus nonbiologic treatment.

For statistical purposes, various groups of disease activity were given numbers: 0 for no disease activity, 1 for mild disease activity, 2 for moderate to severe disease activity.

Statistical analysis. We performed simple descriptive statistic calculations to define whether the category of disease activity had changed, and defined percentage of women by category of disease activity before pregnancy, in each trimester and after delivery. All statistical analyses were carried out with SPSS. Fisher's exact test was used to obtain $\mathrm{p}$ values for an association between worsening of disease activity and discontinuation of biologic treatment and to determine whether there was a significance in improvement/worsening in the various groups. OR and $95 \% \mathrm{CI}$ for the odds of developing any disease activity after discontinuation of biologic treatment were calculated. $P$ value $(\alpha)$ was considered significant at $<0.05$

\section{RESULTS}

The PsA clinics included 107 women of reproductive age. Of them, 25 women had a record of at least 1 pregnancy during their clinical followup and 1 visit within a year after pregnancy, resulting in 35 pregnancies that were reviewed. Thirty-three pregnancies resulted in the birth of live healthy babies. Two pregnancies that were excluded resulted in a pregnancy loss. The women's mean age at pregnancy was 32.5 years. The baseline characteristics of the pattern of articular involvement were oligoarthritis $=9$, polyarthritis $=16$, and combined axial and peripheral involvement $=2$ (no patients with only axial involvement).

In the whole group, there was no significant change in disease activity throughout pregnancy, but the disease activity of $16(48 \%)$ of them worsened during the postpartum period. There was a trend toward aggravation of disease activity during the postpartum period compared to the pre-pregnancy level. A statistically significant aggravation postpartum was shown compared to the third trimester (16 with no disease activity, 9 with mild disease activity, 8 with moderate to severe disease activity in the third trimester, vs 6 with no disease activity, 9 with mild disease activity, and 18 with moderate to severe disease activity postpartum, $\mathrm{p}=0.01$ ).

Table 1 summarizes the number of patients with none, mild, or moderate to severe disease activity during pregnancy in the whole group.

Prior to 21 pregnancies, the patients were treated with biologic agents as follows: infliximab (IFX) 5, adalimumab (ADA) 6, etanercept (ETN) 8, golimumab (GOL) 1, and ustekinumab 1 . The mean duration of treatment with the latest biologic agent close to pregnancy was 34 months. The biologic treatment was discontinued close to pregnancy or during the first trimester in 15 cases, of which 5 were classified as having mild to severe activity prior to pregnancy. A significant worsening in disease activity was observed in this group of patients during pregnancy and postpartum (the number of patients classified as mild to severe changed to 8 , 9 , and 14 during the first and second trimesters and the postpartum period, respectively). There was significant worsening of disease activity in the postpartum period compared with the third trimester in the group that discontinued biologic treatment ( 7 with no disease activity, 4 with mild disease activity, 4 with moderate to severe disease activity in third trimester, vs 1 with no disease activity, 4 with mild disease activity, 10 with moderate to severe disease activity postpartum, $\mathrm{p}=0.03$; Figure 1 ).

On the other hand, there was no significant change in the level of disease activity during pregnancy and in the postpartum period in 6 patients whose biologics [5 tumor necrosis factor (TNF)- $\alpha$ blockers and 1 ustekinumab] were continued beyond the first trimester.

In the group that was not treated with biologics before pregnancy, there was also a trend toward worsening of disease activity in the postpartum period. Interestingly, there

Personal non-commercial use only. The Journal of Rheumatology Copyright $\subset$ $\subset$ 2018. All rights reserved 
Table 1. Disease activity during pregnancy in the whole group $(\mathrm{n}=33)$.

\begin{tabular}{lccccc}
\hline Activity Level & Before & First Trimester & Second Trimester & Third Trimester & Postpartum \\
\hline None & $14(42)$ & $13(39)$ & $12(38)$ & $16(36)$ & $6(18)$ \\
Mild & $9(27)$ & $10(30)$ & $10(29)$ & $9(27)$ & $9(27)$ \\
Moderate to severe & $10(30)$ & $10(30)$ & $11(33)$ & $8(24)$ & $18(54)$ \\
\hline
\end{tabular}

Values are $\mathrm{n}(\%)$.

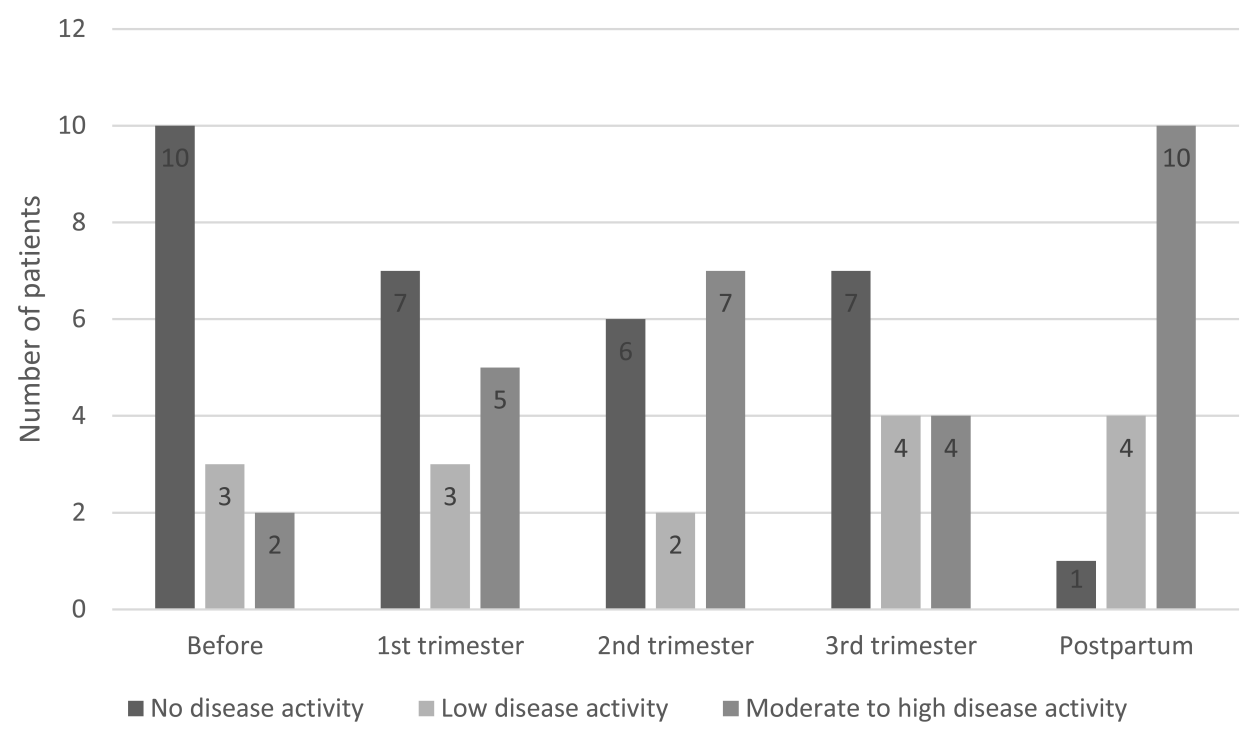

Figure 1. Disease activity in patients in whom biologic treatment was stopped before pregnancy or during first trimester $(n=15)$. An increase in disease activity was observed in patients who stopped biologics before or during the first trimester of pregnancy.

was an improvement in disease activity during pregnancy in this group. Specifically, the proportion of patients with mild to severe disease activity decreased from $85 \%$ close to pregnancy to $69 \%$ in the first and second trimesters and to $58 \%$ in the third trimester, while an increase to $83 \%$ was observed during the postpartum period, a finding not observed in the group that continued the biologic treatment beyond the first trimester.

Comparisons of disease activity before pregnancy and during the postpartum period revealed a significant difference only in the group that discontinued biologic treatment (10 with no disease activity, 3 with mild disease activity, and 2 with moderate to severe disease activity before pregnancy vs 1 with no disease activity, 4 with mild disease activity, and 10 with moderate to severe disease activity postpartum, $\mathrm{p}=$ 0.0001 ). Figure 2 summarizes the changes in disease activity by treatment groups and periods of pregnancy.

Despite the small sample size, we used logistic regression to compare the group with no disease activity and groups with any degree of disease activity. The OR to develop any disease activity after discontinuation of biologic treatment was 7, with a 95\% CI of 0.5-98.6.
Regarding active treatment during pregnancy: only in the group of patients who stopped biologics before or during the first trimester of pregnancy was initiation of treatment with corticosteroids or increase of the dosage of the corticosteroids required. That was done in 6 pregnancies, including intraarticular injections in 2 pregnancies.

\section{DISCUSSION}

The results of our study showed that the disease of women with PsA flares after pregnancy, in accordance with the data reported in a prospective study (15 patients, 20 pregnancies) by Ostensen ${ }^{7}$. Those authors noted that most pregnancies were accompanied by an exacerbation during the 10-week period after delivery. Polachek, et $^{\mathrm{a}} \mathrm{l}^{8}$, in a retrospective study of 29 women with PsA with 42 pregnancies, concluded that the disease activity of $43 \%$ of the patients either worsened or was high but remained the same in the postpartum period. Raychaudhuri, et $a l^{6}$, in a retrospective study of 736 questionnaires filled out by patients with psoriasis without arthritis, reported that 56\% improved and $26.4 \%$ worsened during pregnancy, while there was no significant postpartum worsening of the psoriasis.

Personal non-commercial use only. The Journal of Rheumatology Copyright $\odot$ 2018. All rights reserved. 


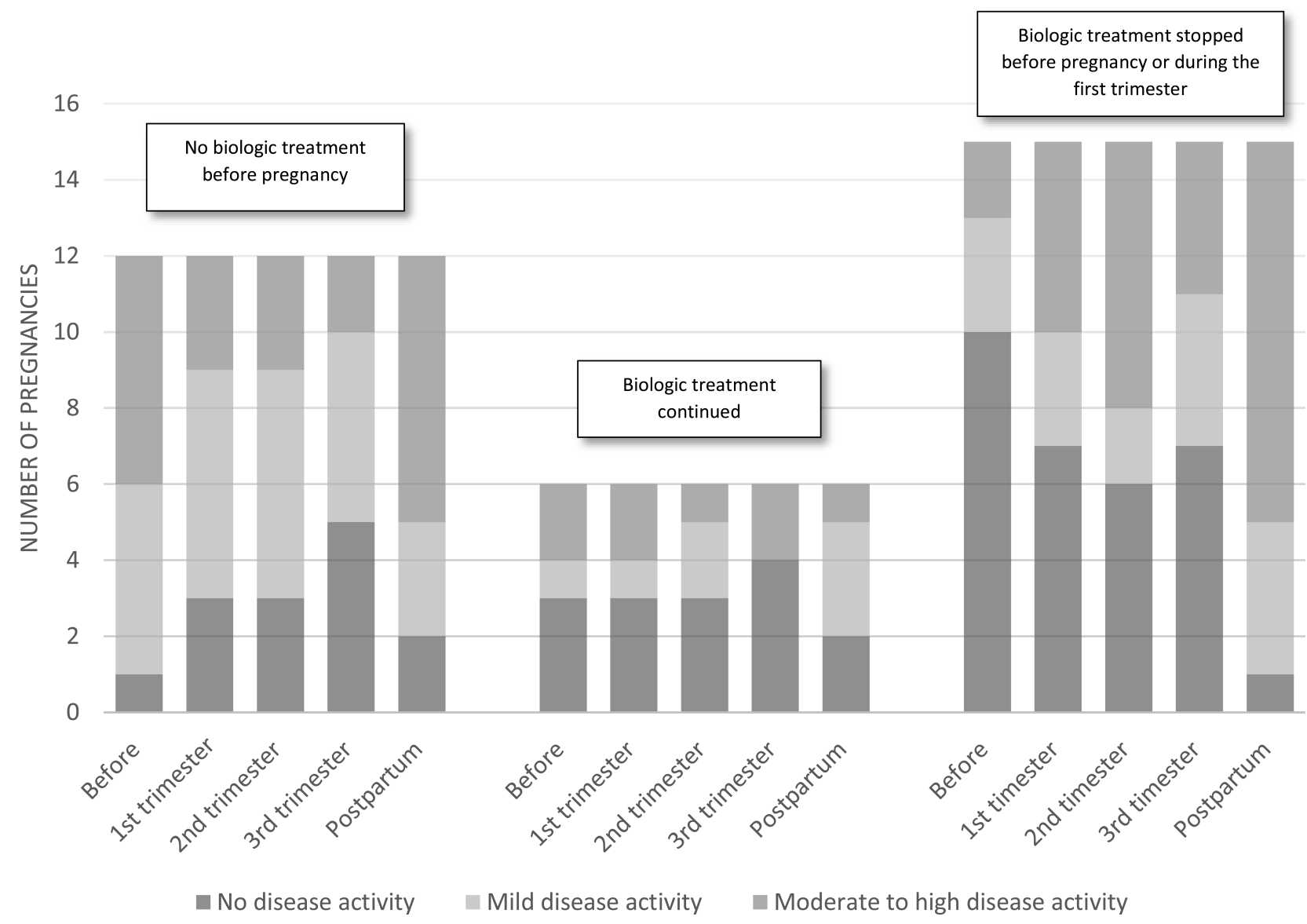

Figure 2. Change in disease activity during pregnancy by groups: no biologic treatment before pregnancy, biologic treatment continued, biologic treatment stopped before pregnancy or during first trimester. In the group not treated with biologics before pregnancy, improvement in disease activity was observed during the pregnancy with worsening in the postpartum period. In the group that continued the biologic treatment, the disease activity was stable. In the group that stopped the biologics, significant worsening of disease activity in the postpartum period was shown.

Although not consistent, different authors have reported a significant increase of at least 1 adverse outcome of pregnancy such as spontaneous abortion, small or large for gestational weight, cesarean delivery, and prematurity ${ }^{9,10}$ among women with psoriasis. On the other hand, a systematic review of observational studies showed no clear evidence of increased adverse outcomes in pregnant women with psoriasis ${ }^{11}$.

Overall, there was no significant change in disease activity throughout pregnancy for our entire study group, while the disease activity of a significant proportion of patients flared during the postpartum period. There were also significant differences between subgroups of nonbiologic-treated patients and biologic-treated patients in whom the treatment was stopped before or during the first trimester of pregnancy, as well as patients in whom biologic treatment was continued throughout the pregnancy. A low level of disease activity and a low probability of flare during pregnancy were well associated with the continuation of therapy with biologics, while stopping them before pregnancy correlated with an increased number of flares.
There were no fetal abnormalities among the infants born to any of our study women. That finding is in agreement with those of some case series in the literature related to biologic treatment of patients with psoriasis during pregnancy. No fetal abnormalities were observed in the offspring of 7 patients from the BIOBADADERM registry. In that report, 10 patients with psoriasis were treated with biologic therapy during gestation: 6 received ustekinumab, 1 received ADA, and 1 received ETN during the initial weeks of pregnancy, and 2 received IFX throughout their pregnancies. All but 2 patients had a normal pregnancy and delivery: 1 had a spontaneous abortion and the newborn of another was a collodion baby whose skin normalized over a period of 2 months ${ }^{12}$.

Notably, IFX, ADA, and ustekinumab are actively transported through the placenta in the same way as natural maternal antibodies, reaching high levels in the blood of newborns after they had been exposed in the late second and third trimesters. Both IFX and ADA have been found in newborns in much higher concentrations than in the 
peripheral blood of their mothers, remaining detectable from 2 to 7 months after birth. ETN, on the other hand, showed considerably less transplacental transport than IgG, while certolizumab pegol (CZP) did not seem to cross the placenta $^{13}$.

There is some concern that the use of biologics that actively cross the placenta during pregnancy could result in immunosuppression in a newborn and increase the risk of infection ${ }^{13}$. One case of a fatal disseminated bacillus Calmette-Guérin infection after regular vaccination in an infant delivered by a mother with Crohn's disease who was treated throughout pregnancy with IFX was reported by Cheent, et $a l^{14}$. European League Against Rheumatism recommendations, however, indicate that IFX and ADA can be continued until gestational week 20, and that ETN and CZP can be continued up to gestational weeks $30-32^{15}$. Therefore, it would appear that those drugs can be used safely throughout pregnancy. As for GOL and ustekinumab, the current evidence does not indicate an increased rate of congenital malformations, but because of the paucity of data, alternative medications should be considered for treatment throughout pregnancy. The main limitation in continuing biologics throughout pregnancy concerns the subsequent administration of live vaccines in newborns exposed to biologic medication during the late second and third trimesters. These vaccines should be postponed until 6 to 7 months of age or until the biological agent is no longer detectable in the infant's circulation. Routine vaccinations with nonlive vaccines appear to be safe, and responses appear to be appropriate ${ }^{16}$.

Our study has several limitations. The retrospective design of the study as well as the relatively small number of patients may have influenced the conclusions. In addition, only the pregnancies that ended with the birth of a live baby were included. This represents a possible bias of the study. However, the main objective was to evaluate the effect of pregnancy on disease activity without focusing on possible adverse outcomes of the pregnancy.

Our results suggest that stopping treatment with biologics, mainly TNF- $\alpha$ blockers before pregnancy, is associated with flare of disease activity during the pregnancy and the postpartum period of women with PsA. It seems that regarding PsA disease activity, it may be recommended to continue treatment with TNF- $\alpha$ blockers during conception and up to the second trimester of pregnancy.

\section{REFERENCES}

1. Helmick CG, Lee-Han H, Hirsch SC, Baird TL, Bartlett CL. Prevalence of psoriasis among adults in the U.S.: 2003-2006 and 2009-2010 National Health and Nutrition Examination Surveys. Am J Prev Med 2014;47:37-45.

2. Persellin RH. The effect of pregnancy on rheumatoid arthritis. Bull Rheum Dis 1976-77;27:922-7.

3. De Man YA, Dolhain RJ, van de Geijn FE, Willemsen SP, Hazes JM. Disease activity of rheumatoid arthritis during pregnancy: results from a nationwide prospective study. Arthritis Rheum 2008;59:1241-8.

4. Clowse ME, Jamison M, Myers E, James AH. A national study of the complications of lupus in pregnancy. Am J Obstet Gynecol 2008;199:127.e1-6.

5. Smyth A, Oliveira GH, Lahr BD, Bailey KR, Norby SM, Garovic VD. A systematic review and meta-analysis of pregnancy outcomes in patients with systemic lupus erythematosus and lupus nephritis. Clin J Am Soc Nephrol 2010;5:2060-8.

6. Raychaudhuri SP, Navare T, Gross J, Raychaudhuri SK. Clinical course of psoriasis during pregnancy. Int J Dermatol 2003; 42:518-20.

7. Ostensen M. Pregnancy in psoriatic arthritis. Scand J Rheumatol 1988;17:67-70.

8. Polachek A, Li S, Polachek IS, Chandran V, Gladman D. Psoriatic arthritis disease activity during pregnancy and the first-year postpartum. Semin Arthritis Rheum 2017;46:740-5.

9. Cohen-Barak E, Nachum Z, Rozenman D, Ziv M. Pregnancy outcomes in women with moderate-to-severe psoriasis. J Eur Acad Dermatol Venereol 2011;25:1041-7.

10. Ben-David G, Sheiner E, Hallak M, Levy A. Pregnancy outcome in women with psoriasis. J Reprod Med 2008;53:183-7.

11. Bobotsis R, Gulliver WP, Monaghan K, Lynde C, Fleming P. Psoriasis and adverse pregnancy outcomes: a systematic review of observational studies. Br J Dermatol 2016;175:464-72.

12. Echeverría-García B, Nuño-González A, Dauden E, Vanaclocha F, Torrado R, Belinchón I, et al. A case series of patients with psoriasis exposed to biologic therapy during pregnancy: the BIOBADADERM Register and a review of the literature. Actas Dermosifiliogr 2017;108:168-70.

13. Mervic L. Management of moderate to severe plaque psoriasis in pregnancy and lactation in the era of biologics. Acta Dermatovenerol Alp Pannonica Adriat 2014;23:27-31.

14. Cheent K, Nolan J, Shariq S, Kiho L, Pal A, Arnold J. Case report: fatal case of disseminated BCG infection in an infant born to a mother taking infliximab for Crohn's disease. J Crohns Colitis 2010;4:603-5.

15. Götestam Skorpen C, Hoeltzenbein M, Tincani A, Fischer-Betz R, Elefant E, Chambers C, et al. The EULAR points to consider for use of antirheumatic drugs before pregnancy, and during pregnancy and lactation. Ann Rheum Dis 2016;75:795-810.

16. Rubin LG, Levin MJ, Ljungman P, Davies EG, Avery R, Tomblyn M, et al. 2013 IDSA clinical practice guideline for vaccination of the immunocompromised host. Clin Infect Dis 2014;58:309-18.

Personal non-commercial use only. The Journal of Rheumatology Copyright (C) 2018. All rights reserved. 